\title{
Long-Term Neurophysiologic Impact of Childhood Sleep Disordered Breathing on Neurocognitive Performance
}

\author{
Stuart F. Quan, M.D. ${ }^{a b}$ \\ Stuart Quan@hms.harvard.edu \\ Kristen Archbold, Ph.D. ${ }^{c}$ \\ Karchbold@nursing.arizona.edu \\ Alan S. Gevins, D.Sc. ${ }^{d}$ \\ Alan@eeg.com \\ James L. Goodwin, Ph.D. ${ }^{a}$ \\ jamieg@arc.arizona.edu
}

a Arizona Respiratory Center, University of Arizona College of Medicine, Tucson, AZ, bDivision of Sleep Medicine, Harvard Medical School, Boston, MA, CPractice Division, University of Arizona College of Nursing, Tucson, AZ, dSAM Technology \& San Francisco Brain Research Institute, San Francisco, CA

\begin{abstract}
Study Objective. To determine the impact of sleep disordered breathing (SDB) in children on neurocognitive function 5 years later.

Design, Setting, and Participants. A subgroup of 43 children from the Tucson Children's Assessment of Sleep Apnea Study (TuCASA) who had SDB (RDI $\geq 6$ events/hour) at their initial exam (ages 6-11 years) were matched on the basis of age (within 1 year), gender and ethnicity (Anglo/Hispanic) to 43 children without SDB (Control, RDI $\leq 4$ events/hour). The Sustained Working Memory Task (SWMT) which combines tests of working memory (1-Back Task), reaction time (Simple Reaction Time) and attention (Multiplexing Task) with concurrent electroencephalographic monitoring was administered approximately 5 years later. Results. There were no differences in performance on the working memory, reaction time and attention tests between the SDB and Control groups. However, the SDB group exhibited lower P300 evoked potential amplitudes during the Simple Reaction Time and Multiplexing Tasks. Additionally, peak alpha power during the Multiplexing Task was lower in the SDB Group with a similar trend in the Simple Reaction Time Task $(p=0.08)$.

Conclusions. SDB in children may cause subtle long-term changes in executive function that are not detectable with conventional neurocognitive testing and are only evident during neuroelectrophysiologic monitoring.
\end{abstract}

\section{Introduction}

There is increasing evidence that childhood sleep disordered breathing (SDB) is associated with neurobehavioral morbidity (1-3). In cross-sectional studies, 
children with SDB are found to have deficits in a variety of neurobehavioral domains including attention, executive function, behavior regulation, alertness, learning and academic performance (3). Treatment of OSA with either tonsillectomy or adenoidectomy often results in resolution or improvement in many of these domains $(4,5)$.

Despite the large amount of data implicating SDB as a causative factor in producing deficits in neurocognition in children, there have been few studies implicating SDB in children as a risk for long-term neurobehavioral morbidity. Several studies have reported that snoring as a surrogate for SDB predicted increased risk for hyperactive behavior (6-8). In addition, in a retrospective analysis, Gozal and Pope (9) reported that low performing middle school students had a greater likelihood of snoring during childhood than their high performing classmates. However, there have been no studies of long-term neurobehavioral morbidity that have used polysomnography (PSG) to document the presence of SDB. Determining whether long-term or permanent deficits in neurocognition occur as a result of SDB will be important in timing of treatment intervention in these children.

In the present study, a subset of the Tucson Children's Assessment of Sleep Apnea Study (TuCASA) underwent additional cognitive neurophysiological testing to determine whether SDB documented during childhood was a risk factor for deficits 5 years later. We hypothesized that children with SDB would exhibit subtle abnormalities during these neurophysiologic tests.

\section{Materials \& Methods}

Subjects. The Tucson Children's Assessment of Sleep Apnea study (TuCASA) was a longitudinal cohort established to investigate the correlates and natural history of childhood sleep disordered breathing. Recruitment and overall study methods have been previously described $(10,11)$. In brief, the TuCASA cohort consisted of healthy school-aged children that were enrolled in a large urban school district in the Southwest United States. With the cooperation of their respective elementary schools, parents of the students were asked to complete a brief screening questionnaire and to provide contact information if they wanted to see if their child was eligible for the study. Those who qualified were then studied using a single overnight unattended in-home PSG along with completion of a questionnaire regarding their sleep habits. TuCASA initially recruited 503 participants (ages 6 11) who had their PSGs recorded between the years of 2000 and 2004. Approximately 5 years later, the study attempted to contact the same participants and was successful in restudying 319 children who had valid PSGs for both the baseline and follow-up time points. From this group, we selected 2 groups of children based on their respiratory disturbance index measured from the PSG performed during their baseline exam cycle. No children had received continuous positive airway pressure treatment for SDB. The first group (SDB) consisted of children with SDB as defined by a respiratory disturbance index $(\mathrm{RDI})>6$ /hour. 
The second group (Control) was children without SDB as defined by a RDI < 4/hour. Each child in the Control group was matched to a child in the SDB group on the basis of age (within 1 year), gender and ethnicity. This resulted in a study cohort of 43 pairs of children.

All methods used to recruit subjects and to collect the present data set were approved both by the University of Arizona Human Subjects Committee and the Tucson Unified School District Research Committee. In all cases, we obtained written informed consent from the parents, and assent from the children.

Study Design. Children from both groups were asked to return to the TuCASA sleep laboratory to undergo the Sustained Working Memory Test (SWMT) which was adapted for use in children. The SWMT is an automated cognitive neurophysiological test that combines cognitive test performance measures with electroencephalograhic (EEG) measures. It has been validated in distinguishing cognitive performance in subjects who have ingested alcohol, caffeine, diphenhydramine and who have been sleep deprived $(12,13)$. These studies were performed on a day separate from any other testing done for TuCASA.

Polysomnography. In both the baseline and follow-up examinations, children underwent unattended, nocturnal home PSG using the Compumedics PS-2 system (Abbotsford, Victoria, Australia) $(10,11)$. The following signals were obtained: $\mathrm{C}_{3} / \mathrm{A}_{2}$ and $\mathrm{C}_{4} / \mathrm{A}_{1} \mathrm{EEG}$, right and left electrooculogram, a bipolar submental electromyogram, thoracic and abdominal displacement (inductive plethysmography bands), airflow (nasal/oral thermocouple), nasal pressure, electrocardiogram (single bipolar lead), snoring (microphone attached to a vest), body position ( $\mathrm{Hg}$ gauge sensor), pulse oximetry (Nonin, Plymouth, MN) and ambient light (sensor attached to the vest to record on/off). Using Compumedics W-Series Replay, $v 2.0$, release 22, sleep stages were scored according to Rechtschaffen and Kales criteria (14). The RDI was defined as the number of respiratory events (apneas and hypopneas) per hour of the total sleep time irrespective of any associated oxygen desaturation or arousal. Studies with less than 4 hours of scorable oximetry were classified as failed studies and were repeated if the participant consented. Central apneas were scored if both airflow and thoracoabdominal effort were absent. However, central events that occurred after movement were not included. Obstructive apneas were identified if the airflow signal decreased to below $25 \%$ of the "baseline amplitude". Hypopneas were scored if the magnitude of any ventilation signal decreased below approximately $70 \%$ of the "baseline" amplitude, as described previously (15).

Body Mass Index Computation. Height and weight were collected on a platform scale. BMI was calculated $\mathrm{kg} / \mathrm{m}^{2}$, and percentile of BMI adjusted for age, sex and ethnicity was calculated with a standardized data-analysis program from the Centers for Disease Control (http://www.cdc.gov/nccdphp/dnpao/growthcharts/resources/sas.htm ). 
Wechsler Abbreviated Scale of Intelligence (WASI). The WASI (16) is nationally standardized intelligence test which is linked to the Wechsler Intelligence Scale for Children ${ }^{\circledR}$-Fourth Edition (WISC-IV®). It was administered in TuCASA as part of an overall neurocognitive test battery within several weeks of the SWMT.

Sustained Working Memory Test. The SWMT (17) consists of a brief 25 minute computerized test consisting of two blocks of an attentional multiplexing task, an easy and a more difficult version of a spatial n-back working memory task, and eyes open and eyes closed resting tasks. The test is designed for concurrent EEG recording. Data collected included EEG and evoked potential (EP) signals, as well as task performance measures. All subjects were trained how to perform each task the same day the test was administered.

In the attentional multiplexing task (MT), the participants were required to monitor multiple stimuli as they changed shape, color, and pattern, and to sort each object into a bin based on its relevant features. This task adapted to an individual's ability level; task difficulty increases if performance exceeds a pre-defined threshold, and decreases if performance falls below the threshold. Each MT block lasted approximately 3.5 minutes. The working memory (WM) test consisted of a 3.5 minute spatial 1-back task, in which participants compared the location of the dot stimulus on each trial to that on the immediately preceding trial. A simple reaction time (SRT) test with the same stimulus and response characteristics also was administered as a control task. Resting EEG was also recorded for 1.5 min with eyes open and $1.5 \mathrm{~min}$ with eyes closed.

EEGs were recorded from seven scalp locations (Fz, F3, F4, Cz, Pz, P3, P4) positioned via a nylon electrode cap and referenced to linked mastoids. This montage was designed to include adequate spatial representation of the signal features of primary interest as defined by prior high-resolution EEG studies of the working memory tasks used herein (18). Potentials generated by eye movements and blinks were recorded by electrodes positioned above and at the outer canthus and superior orbital ridge of each eye. The resulting data were digitally high-pass filtered at $0.5 \mathrm{~Hz}$. EEG was recorded continuously during task performance and during passive resting conditions. Electrode impedances were kept $<5 \mathrm{~K} \Omega$ for the references and $<20 \mathrm{~K} \Omega$ on all other channels.

Automated artifact-detection and artifact-decontamination filters were used to minimize contaminants induced by eye movement and other physiologic and instrumental sources. All data were then visually inspected, and any residual contaminants were excluded from further analysis.

SWMT Data Analysis. To assess neurophysiological measures between the SDB and Control groups, EEG power spectra and EPs were calculated. Power spectra were computed from all artifact-free EEG for each task block and converted to $\mathrm{dB}$ power with a log10 transformation. To calculate EPs, trials were averaged in $1.2 \mathrm{~s}$ epochs beginning $0.2 \mathrm{~s}$ before stimulus onset. EP peak amplitudes were measured 
relative to the mean amplitude in the prestimulus interval.

A number of prior studies have served to identify spectral features of the EEG that are sensitive to task-difficulty manipulations in the types of working memory tasks used in the SWMT Exam (18-20). Based on such previous findings, a number of such sensitive EEG and EP signals were compared between the SDB and Control groups. EEG alpha power was measured as the maximum power in a $2 \mathrm{~Hz}$ band between 8- to $12-\mathrm{Hz}$ in all tasks. Amplitude of the P300 (measured in a $100 \mathrm{~ms}$ window centered on the largest positive peak between 250 and $520 \mathrm{~ms}$ at $\mathrm{Pz}$ ), and slow-wave EPs (measured in a $250 \mathrm{~ms}$ window centered on the largest positive peak between 250 and $650 \mathrm{~ms}$ ) were computed in the SRT and 1-back WM tasks. Because of the nature of the MT task, EPs were calculated relative to the onset of the visual feedback that immediately follows a correct or incorrect response. A P300 was measured in the MT as the largest positive peak occurring 200-450 ms after the feedback.

Statistical Methods. Potential differences in the characteristics of the study population between the SDB and Control groups were evaluated using Students' unpaired t-test or linear correlation. Inasmuch as intelligence is a significant factor in determining performance on neurocognitive tests, analysis of covariance was used to compare performance on the various components of the SWMT between groups while controlling for intelligence as assessed by the WASI. Other covariates were included in the models if significant on univariate analyses. Data were analyzed with IBM SPSS Version 20 (http://www-

$01 . \mathrm{ibm} . \mathrm{com} / \mathrm{software} /$ analytics/spss/) and are presented as mean $\pm \mathrm{SE}$.

\section{Results}

In Table 1 is shown the characteristics of the children in this study.

Table 1. Characteristics of the Study Population

\begin{tabular}{|l|c|c|}
\hline & Control & SDB \\
\hline No. of Pairs & \multicolumn{2}{|c|}{43} \\
\hline $\mathrm{n}(\%)$ Boys/n(\%) Girls & $25(58 \%) / 18(42 \%)$ & $25(58 \%) / 18(42 \%)$ \\
\hline $\begin{array}{l}\mathrm{n}(\%) \text { Anglos/n(\%) } \\
\text { Hispanics }\end{array}$ & $32(74 \%) / 11(26 \%)$ & $32(74 \%) / 11(26 \%)$ \\
\hline Age at $1^{\text {st }} P S G$ & $8.7 \pm 1.6$ & $8.9 \pm 1.8$ \\
\hline Age at $2^{\text {nd }} P S G$ & $13.3 \pm 1.5$ & $13.6 \pm 1.6$ \\
\hline Age at SWMT & $13.5 \pm 1.4$ & $13.7 \pm 1.6$ \\
\hline RDI $1^{\text {st }} P S G$ & $2.5 \pm 0.1$ & $9.5+0.6^{a}$ \\
\hline RDI $2^{\text {nd }} P S G$ & $2.0 \pm 1.7$ & $3.1 \pm 2.5^{b}$ \\
\hline $\begin{array}{l}\text { Standardized BMl at } 1^{\text {st }} \\
\text { PSG }\end{array}$ & $53.0 \pm 4.8$ & $67.5 \pm 5.1^{\mathrm{a}}$ \\
\hline $\begin{array}{l}\text { Standardized BMl at } 2^{\text {nd }} \\
\text { PSG }\end{array}$ & $57.1 \pm 5.0$ & $70.1 \pm 5.0^{\mathrm{a}}$ \\
\hline WASI IQ at SWMT & $108.0 \pm 1.7$ & $109.1 \pm 2.1$ \\
\hline
\end{tabular}

Data shown as Mean+SE

Standardized BMIs have been converted to percentile

a $p<0.01$ No SDB vs. SDB

${ }^{b} p<0.05$ No SDB vs. SDB 
Boys and Anglos comprised the majority of the study cohort. No children had undergone an adenotonsillectomy at the time of the $1^{\text {st }} \mathrm{PSG}$, and only 2 had this procedure during the time interval before the $2^{\text {nd }}$ PSG. As defined by the study design, there were no differences between the SDB and Control groups with respect to age, the time of the 1 st or $2^{\text {nd }}$ PSG, or at time of SWMT. Additionally, as dictated by the study design, RDI at the 1 St PSG was significantly greater in the SDB group as was the BMI and the standardized BMI (sBMI). The RDI at the $2^{\text {nd }}$ PSG also was higher in the SDB group. However, RDI decreased in both groups over the time period from the 1 st to the $2^{\text {nd }}$ PSG. Seven children had SDB on both PSGs and 2 children developed SDB over the study interval. In the SDB group, the mean RDI at the $2^{\text {nd }}$ PSG was below the RDI threshold used to define the Control group at the baseline examination (1 1 st PSG). Significant, but weak negative correlations were observed between sBMI and some of the EEG and evoked potential components of the SWMT [1 back peak alpha, $r=-.23$, $\mathrm{p}=0.03$; Multiplex Block 1 Alpha Power, $r=-.25, p=0.02$; Multiplex Block 2 Alpha Power, $r=-.21, p=0.05$; eyes closed peak alpha, $r=-.29, p<0.01$; eyes open delta theta power, $r=-.25, p=0.02]$. Overall, the WASI indicated that the cohort was above average in intelligence and there were no differences between the 2 groups. However, there was considerable heterogeneity within the overall cohort (Minimum WASI: 77; Maximum WASI: 138).

The results of various components of the SWMT are shown in Table 2.

Table 2: Sustained Working Memory Test Performance According to Sleep Disordered Breathing Status

\begin{tabular}{|c|c|c|c|c|}
\hline \multirow[t]{2}{*}{ SWMT Components ${ }^{3}$} & \multicolumn{2}{|c|}{ Control } & \multicolumn{2}{|c|}{ SDB } \\
\hline & Mean & SE & Mean & SE \\
\hline SRT \% Correct & 97.3 & 0.6 & 97.1 & 0.6 \\
\hline SRT Mean Reaction Time (ms) & 341.7 & 13.0 & 353.1 & 20.2 \\
\hline 1 Back \% Correct & 83.3 & 2.0 & 84.9 & 1.6 \\
\hline 1 Back Mean Reaction Time (ms) & 664.7 & 32.1 & 650.6 & 32.9 \\
\hline Multiplex Block 1 Mean Composite Score & 121.9 & 2.0 & 122.3 & 2.2 \\
\hline Multiplex Block 2 Mean Composite Score & 124.5 & 1.9 & 129.8 & 1.9 \\
\hline SRT P300 Amplitude (AV) & 7.4 & 0.6 & 6.4 & 0.6 \\
\hline 1 Back P300 Amplitu de ( $\Delta \mathrm{V})$ & 7.6 & 0.6 & 7.7 & 0.6 \\
\hline SRT Slow Wave Amplitude ( $\Delta \mathrm{V}$ ) & 1.9 & 0.2 & 1.4 & 0.2 \\
\hline 1 Back Slow Wave Amplitude $\left(\Delta V^{\mathrm{b}}\right.$ & 3.7 & 0.4 & 3.0 & 0.3 \\
\hline SRT Peak Alpha Power (dB) ${ }^{\circ}$ & 27.7 & 0.7 & 26.5 & 0.6 \\
\hline 1 Back Peak Alpha Power (dB) & 27.2 & 0.6 & 26.1 & 0.5 \\
\hline Multiplex P300 Amplitude $\left(\Delta V^{d}{ }^{d}\right.$ & 4.2 & 0.4 & 3.3 & 0.3 \\
\hline Multiplex Block 1 Peak Alpha Power (dB)e & 24.3 & 0.4 & 23.0 & 0.3 \\
\hline Multiplex Block 2 Peak Alpha Power $(\mathrm{dB})^{\dagger}$ & 24.5 & 0.4 & 23.3 & 0.3 \\
\hline Eyes Closed Peak Alpha Power (dB) & 35.0 & 0.7 & 34.0 & 0.7 \\
\hline Eyes Open Slow Eye Movement Alpha Power (dB) & 38.8 & 0.8 & 37.7 & 0.7 \\
\hline Eyes Open Delta Theta Power (dB) & 27.7 & 0.4 & 27.5 & 0.3 \\
\hline Resting Alpha Power Divergence Ratio & 0.44 & .02 & 0.43 & .02 \\
\hline
\end{tabular}

aN=43 in both No SDB and SDB groups except for SRT P300 Amplitude, 1 Back P300 Amplitude, Multiplex P300 Amplitude and Eyes Open Slow Eye Movement Alpha Power Tests where $N=42$ in the No SDB group

${ }^{b} p=0.05$ No SDB vs. SDB controlling for WASI IQ

${ }^{c} p=0.08$ No SDB vs. SDB controlling for WASI IQ

${ }^{d} p=0.06$ No SDB vs. SDB controlling for WASI IQ and standardized BM

$e_{p}=0.02$ No SDB vs. SDB controlling for WASI IQ and standardized BMI

${ }^{t} p=0.04$ No SDB vs. SDB controlling for WASI IQ and standardized BMI 
Increased slow eye movement, increased delta/theta band power, and decreased eye closed to eyes open alpha power ratio are neurophysiological indicators of decreased alertness. No differences between the SDB and Control groups were observed for any of these alertness measures. Similarly, there were no differences with respect to either \% items correct or reaction time for the Simple Reaction Time, 1 Back or Multiplexing Tasks. However, the 1 back slow wave amplitude was lower in the SDB Group, and there was a strong trend for the P300 evoked potential amplitude during the Multiplexing Task $(p=0.06)$ and peak alpha power during the Simple Reaction Time Task $(p=0.08)$ to be lower as well. In addition, peak alpha power during the both blocks of the Multiplexing Task was lower in the SDB Group.

Additional analyses were performed to determine whether performance on the SWMT was related to the presence of SDB at the time of the $2^{\text {nd }} P S G$. No differences were observed between children who had SDB on the $2^{\text {nd }} P S G$ and those who did not.

\section{Discussion}

In this study, we have demonstrated that after approximately 5 years, several conventional measures of executive function were not different between children with and without SDB. However, neuroelectrophysiologic assessments recorded during task performance were able to distinguish between these 2 groups. These data suggest that SDB in children can have a long-term, albeit subtle impact on neurocognition in children.

Two important domains of executive function are attention and working memory. In our study these were assessed using a simple reaction time task, a multiplexing task and a 1 back working memory task. Although we did not observe that children with SDB had worse performance in either of these domains, previous cross-sectional studies in children have found deficits using a variety of instruments (3). However, many of these studies assessed children derived from clinic populations. In addition, none determined if there was any impact on long-term performance.

The principal finding from our study is that peak alpha power during the multiplexing and simple reaction time tasks was lower in the SDB group. Alpha power reduction is generally considered a marker of cortical activation. Thus, during task performance, peak alpha power should decline as a function of the amount of effort needed to accomplish a given task (21). It is possible that SDB children in this study may have expended more effort to maintain task performance, as evidenced by a lower alpha power. Using the SWMT, similar findings have been observed after marijuana smoking (22).

Similar to the differences in peak alpha power between our SDB and Control groups, we also observed that the P300 evoked potential amplitude during the 
multiplexing task and the slow wave evoked potential amplitudes during the 1 back task were lower in the SDB group. These evoked potential components are thought to represent aspects of memory encoding, manipulation and retrieval (23). Thus, these data suggest that children with SDB may experience subtle long-term impairment in memory function.

There are several possible explanations of why we did not observe any overt deficits in executive function in children with SDB. First, there was a significant improvement in the RDI in the approximately 5 year interval between the 1st PSG and the testing of these children. Thus, in many of the children, remission of their SDB occurred leading to a reduction in any possible impact of SDB on neurocognition. This would support the contention that overt neurocognitive deficits produced by SDB in school-aged children resolve if SDB improves. Second, the cohort overall had above average intelligence. It is plausible that any impact of SDB would be more evident in those who have less cognitive abilities. Third, it is possible that the 1-back working memory task used in the study was not sufficiently difficult to expose any underlying impairments in executive function. Finally, there is the possibility that inherent cognitive reserve is mitigating the impact of SDB. The cognitive reserve theory postulates that individual differences in how the brain processes tasks may prevent greater insult by using preexisting cognitive processes or by recruiting compensatory ones before there is a detrimental impact on performance (24). Inasmuch as children have the potential for a high amount of neural plasticity, this explanation may be highly relevant in children with SDB.

Our study is not without some important limitations. First, classification of these children into Control and SDB groups was done without regards to desaturation during apnea and hypopnea events. Thus, the impact of oxygen desaturation cannot be assessed. It is unlikely, however, that this is a major confounder because significant oxygen desaturation below $90 \%$ was uncommonly observed in these children. Second, there may have been misclassification of children into the SDB and Control groups especially at the cutpoint boundary. We believe this is less likely because by setting the Control cutpoint at $\leq 4$ events per hour and the SDB cutpoint at $\geq 6$ events per hour, there would have been less risk of misclassification. Third, we did not use intelligence as a factor in assigning children to the 2 groups. However, we believe this had little impact on our results because there was no difference between the groups on the WASI, and we controlled for intelligence in the analyses. Finally, we also observed that body mass index was negatively correlated with performance on some of the SWMT components. Others have found that neurocognitive performance may be negatively associated with obesity (25). However, most studies have been cross-sectional and thus the directionality and causal mechanisms of this association are unclear. Nevertheless, it is unlikely that our results can be explained by this association inasmuch as we controlled for BMI in our analyses. Despite these aforementioned limitations, our study is the only one to our knowledge that has simultaneously assessed neuroelectrophysiologic function during performance of executive function testing 
in children thus demonstrating its feasibility and potential for acquiring unique information.

In conclusion, SDB in children has the potential to result in subtle long-term detrimental effects in executive function that are not detectable with conventional neurocognitive testing, but may be evident with simultaneous neuroelectrophysiologic monitoring. These data emphasize the importance of recognizing and treating SDB in children in order to prevent possible long-term consequences in neurocognitive function.

\section{Acknowledgements}

TuCASA was supported by HL62373. In addition, development of the SWMT was supported by grants from the National Institute of Neurological Diseases and Stroke, The National Institute of Mental Health, The Air Force Research Laboratory and The Office of Naval Research.

Conflicts of Interests: The authors do not have any conflicts of interest to disclose.

\section{References}

1. Owens JA. Neurocognitive and behavioral impact of sleep disordered breathing in children. Pediatr Pulmonol. 2009;44(5):417-422. [CrossRef] [PubMed]

2. Grigg-Damberger M, Ralls F. Cognitive dysfunction and obstructive sleep apnea: from cradle to tomb. Curr Opin Pulm Med. 2012;18(6):580-587. [CrossRef] [PubMed]

3. Beebe DW. Neurobehavioral morbidity associated with disordered breathing during sleep in children: a comprehensive review. Sleep. 2006;29(9):1115-1134. [PubMed]

4. Giordani B, Hodges EK, Guire KE, et al. Changes in neuropsychological and behavioral functioning in children with and without obstructive sleep apnea following Tonsillectomy. J Int Neuropsychol Soc. 2012;18(2):212-222. [CrossRef] [PubMed]

5. Ikeda FH, Horta PA, Bruscato WL, Dolci JE. Intellectual and school performance evaluation of children submitted to tonsillectomy and adenotonsillectomy before and after surgery. Braz J Otorhinolaryngol. 2012;78(4):17-23. [PubMed]

6. Chervin RD, Ruzicka DL, Archbold KH, Dillon JE. Snoring predicts hyperactivity four years later. Sleep. 2005;28(7):885-890. [PubMed]

7. Ali NJ, Pitson D, Stradling JR. Natural history of snoring and related behaviour problems between the ages of 4 and 7 years. Arch Dis Child. 1994;71(1):74-76. [PubMed]

8. Urschitz MS, Eitner S, Guenther A, et al. Habitual snoring, intermittent hypoxia, and impaired behavior in primary school children. Pediatrics. 2004;114(4):10411048. [CrossRef] [PubMed]

9. Gozal D, Pope DW,Jr. Snoring during early childhood and academic 
performance at ages thirteen to fourteen years. Pediatrics. 2001;107(6):13941399. [CrossRef] [PubMed]

10. Goodwin JL, Enright PL, Kaemingk KL, et al. Feasibility of using unattended polysomnography in children for research--report of the Tucson Children's Assessment of Sleep Apnea study (TuCASA). Sleep. 2001;24(8):937-944. [PubMed]

11. Goodwin JL, Vasquez MM, Silva GE, Quan SF. Incidence and Remission of Sleep-Disordered Breathing and Related Symptoms in 6- to 17-Year Old Children-The Tucson Children's Assessment of Sleep Apnea Study. J Pediatr. 2010; [CrossRef] [PubMed]

12. Gevins A, McEvoy LK, Smith ME, et al. Long-term and within-day variability of working memory performance and EEG in individuals. Clin Neurophysiol. 2012;123(7):1291-1299. [CrossRef] [PubMed]

13. Smith ME, McEvoy LK, Gevins A. The impact of moderate sleep loss on neurophysiologic signals during working-memory task performance. Sleep. 2002;25(7):784-794. [PubMed]

14. Rechtschaffen A, Kales A. A Manual of Standardized Terminology,

15. Techniques and Scoring System for Sleep Stages of Human Subject. Washington, D.C.: US Government Printing Office, National Institute of Health Publication, 1968

16. Kaemingk KL, Pasvogel AE, Goodwin JL, et al. Learning in children and sleep disordered breathing: findings of the Tucson Children's Assessment of Sleep Apnea (tuCASA) prospective cohort study. J Int Neuropsychol Soc. 2003;9(7):1016-1026. [CrossRef] [PubMed]

17. Wechsler D. Wechsler Abbreviated Scale of Intelligence. San Antonio, TX: Psychological Corp., 1999

18. Gevins A, Smith ME, McEvoy LK, et al. A cognitive and neurophysiological test of change from an individual's baseline. Clin Neurophysiol. 2011;122(1):114120. [CrossRef] [PubMed]

19. Gevins A, Smith ME, McEvoy L, Yu D. High-resolution EEG mapping of cortical activation related to working memory: effects of task difficulty, type of processing, and practice. Cereb Cortex. 1997;7(4):374-385. [CrossRef] [PubMed]

20. Gevins A, Cutillo B. Spatiotemporal dynamics of component processes in human working memory. Electroencephalogr Clin Neurophysiol. 1993;87(3):128-143. [CrossRef] [PubMed]

21. McEvoy LK, Smith ME, Gevins A. Dynamic cortical networks of verbal and spatial working memory: effects of memory load and task practice. Cereb Cortex. 1998;8(7):563-574. [CrossRef] [PubMed]

22. Gevins A, Smith ME, McEvoy LK. Tracking the cognitive pharmacodynamics of psychoactive substances with combinations of behavioral and neurophysiological measures. Neuropsychopharmacology. 2002;26(1):27-39. [PubMed] [PubMed]

23. Hart CL, Ilan AB, Gevins A, et al. Neurophysiological and cognitive effects of smoked marijuana in frequent users. Pharmacol Biochem Behav. 2010;96(3):333-341. [CrossRef] [PubMed] 
24. Polich J. Updating P300: an integrative theory of P3a and P3b. Clin Neurophysiol. 2007;118(10):2128-2148. [CrossRef] [PubMed]

25. Stern Y. Cognitive reserve. Neuropsychologia. 2009;47(10):2015-2028. [CrossRef] [PubMed]

26. Liang J, Matheson BE, Kaye WH, Boutelle KN. Neurocognitive correlates of obesity and obesity-related behaviors in children and adolescents. Int J Obes (Lond). 2013 Aug 5. [Epub ahead of print] [CrossRef] [PubMed] 